\title{
Making History Relevant: The Case of Computing
}

\author{
Gauthier van den Hove \\ CWI, Amsterdam, Netherlands \\ G.van.den.Hove@ cwi.nl
}

\begin{abstract}
We investigate the motives to practice history, and the role that history could play for a scientific discipline. We consider these questions successively from three interrelated points of view: "history: why?" (\$1), "history: for whom?" (§ 2), and "history: how?" (§3). Only the second of these sections is specific to the field of computing; the two other ones are more general, and could probably be applied to other fields as well. Needless to say, the responses that we propose are elements rather than definitive answers; the author also apologizes in advance if these reflections turn out to be nothing but platitudes: he was not trained as a historian, and it is very well possible that these three questions have already received more convincing answers elsewhere.
\end{abstract}

Keywords: History, computing, historical and ahistorical disciplines.

\section{$1 \quad$ History: Why?}

If one considers the relation that the fields of human knowledge maintain with their own history, there seems to exist a dichotomy between two kinds of disciplines. In philosophy or in arts for example, the detailed study of the history of the field is seen as something of great importance. It is even a prerequisite to be competent in these fields, and students typically spend hours and days studying the achievements of the masters of the past, for example Plato's Meno or Beethoven's Symphonies. On the contrary, in mathematics or in the field of computing, history is most often seen as something of little or no importance: a student in mathematics for instance will not spend a single minute reading Euclid's Elements, even if he uses the results discovered by Euclid daily, and likewise a student in a computer science program will not study the details of, say, the Multics operating system, even if some of the ideas and techniques introduced in Multics are widely used nowadays. All fields of human knowledge can, apparently, be separated in two according to that criterion: is the study of the field's history seen, by the participants of that field, as something essential or not?

This distinction between "historical" and "ahistorical" disciplines is of course not our own discovery: it corresponds for example to the opposition between the "two cultures," literary and scientific, described by C. P. Snow in his famous article and lecture. ${ }^{1}$ However, while the lack of interest for history is clearly a distinctive trait of

1 Snow, C. P., The Two Cultures, The Two Cultures and The Scientific Revolution. 
the vast majority of contemporary scientists, one could argue that there are exceptions to this rule, for instance A. Weil in mathematics. This single example suffices to demonstrate that it is not clear that sciences are, by nature, ahistorical disciplines. In other words, it shows that it is necessary to question our distinction and its validity ( $§ 1.1)$ before trying to derive a few conclusions ( $\$ 1.2$ and $\S 1.3)$.

\subsection{On the Difference between Historical and Ahistorical Disciplines}

It is immediately evident that what happened in the past is not less important for ahistorical disciplines than for historical ones: scientists build upon what has already been achieved by their predecessors, and do not start over again as if nothing had been done before them. This was already clear in the two examples above: the results discovered by Euclid and the techniques introduced in Multics are still used today. What makes the difference between a historical and an ahistorical discipline is thus not that what is dated is considered as outdated for the latter and not for the former. The difference is, rather, that they bear a different relation to the documents in which the results have first been expounded or realized. In philosophy or in arts for example it is necessary to study Kant's Critique of Pure Reason to understand it or Bach's Goldberg Variations to understand them, and teachers will advise students to distrust textbooks and to read the original documents as much as possible. On the contrary, in mathematics or in the field of computing, the older results have been absorbed in later works, and teachers will not advise students to read to original documents, but instead to rely on textbooks in which all the important results are presented synthetically. Even more so, should a student try to read Euler's Introduction to Analysis of the Infinite or to study the details of the ITS operating system for instance, most if not all teachers would urge her not to do so, explaining that it would be a terrible waste of time, or that it is worthless to know the details of these works, or even that studying them could cause confusion.

The difference in the relations with the original documents is more precise than the still vague opposition between historical and ahistorical disciplines with which we started. In turn, it leads us to try to understand its cause.

A first reason that could be proposed is that literary disciplines do not aim, contrary to scientific disciplines, at understanding reality, but only at understanding and commenting these documents. It is easy to see that this reason is not a good one: there is, at the very least, no general agreement in literary disciplines that their only aim is to understand documents from the past, independently of their relation to reality. One would not study Hegel's Elements of the Philosophy of Right, for example, only to understand what a particular man thought in 1820 , but to understand modern political systems better.

A second possible reason is that it seems that there is no unity in literary disciplines, contrary to scientific ones. A sign of this is that it is common, in literary disciplines, to describe oneself as a "Heideggerian" or as an "Aristotelian" for instance, that is to say, to make oneself a disciple of a certain master, whereas in sciences no one would claim to be a "Newtonian" or an "Einsteinian." This division of literary disciplines in particular schools of thought, each of them being in a close 
relation with a given master which it considers as a reference, could explain that these founder's creations are studied in detail, because each participant of the field has to situate himself within the various traditions and to justify his choice. This reason seems a bit more convincing than the previous one, but it is, again, not a good one. There are schools of thought in sciences as well: for example, logicism, intuitionism and formalism are three different ways of founding mathematics. More generally, even if the different traditions are not as apparent in sciences as they are in literary disciplines, because they are not named after their founders, it is clear that they exist; their existence becomes the most apparent, as has been shown by T. S. Kuhn, when they enter in conflict with each other. ${ }^{2}$

A third possible reason to explain the different relationships with original documents is that literary works seem to be more personal than scientific contributions: Schubert's music for example could not have been written by anyone else than Schubert himself, whereas Heisenberg's contributions to physics could in principle have been discovered by someone else. This could explain that it is important to study the original documents in literary disciplines, both because they contain something unique that cannot be synthesized, and because one should learn to create something that is original, contrary to scientific disciplines in which one should contribute something to a larger edifice. This argument seems again better than the previous one, but it is still not a good one. Many literary works would not be described by their author as a creation, but rather as something they have discovered or received. Moreover, sciences are also a creative endeavour in which the scientist's personality plays a role. A. M. Turing gave for instance the first convincing definition of computability in 1936; given that a number of other scientists had attempted to define it earlier without success, it is very unlikely that his contribution could have been made by someone else.

The three arguments we have examined have shown to be unconvincing to justify the difference between disciplines in which studying the original documents is considered important and those in which it is not. Discussing every possible argument would of course be an endless task. We therefore have to reconsider the same question from another point of view, by looking at the inverse question, namely: are literary disciplines historical by nature, that is, is the study of original documents something essential in these disciplines? It turns out that the answer to that question is negative: the study of original documents was not always seen as something important in these disciplines, which means that they have become historical at some point. Descartes or Kant, for example, were apparently very mildly interested in referring to original documents. It is thus not only incorrect that sciences are by nature ahistorical; it is also incorrect that literary disciplines are by nature historical.

This conclusion leads us to a new question: why did literary disciplines became historical, that is, why did the study of original documents became one of their important traits? The main motivations behind this change seem to be a consciousness that studying the past is a source of inspiration $(\S 1.2)$ and a condition of progress $(\S 1.3)$.

2 Kuhn, T. S., The Structure of Scientific Revolutions. 


\subsection{History as a Source of Inspiration}

Stating that history is a source of inspiration seems a rather vague idea, particularly for scientific disciplines; it is thus necessary to explain in more detail what it means. Thucydides, who is considered as the first historian, already explained that history is useful for "those who want to have a clear view of what happened in the past and what - the human condition being what it is - can be expected to happen again some time in the future in similar or much the same ways." ${ }^{3}$ This makes it clear that the aim of history was, right from the start, to shed some clarity not only on past events, but also on current and future ones. What Thucydides had in mind are politics: his detailed study of a specific political event was therefore not meant to satisfy a mere curiosity, but he conceived it as a contribution to the culture of those who are involved in politics, to serve as an inspiration for their choices and acts, by making them aware of a number of constant laws of the "human condition." These laws are not explicitly stated, because it is a part of the reader's task to understand them; they are only illustrated by concrete examples. In other words, his History of the Peloponnesian War is a case study in politics.

Thucydides' approach is applicable, mutatis mutandis, to scientific disciplines. One could try to use it to identify the qualities that make a good mathematician or a good physicist for instance; however, such questions are not, strictly speaking, scientific ones, and are subject to too much uncertainty to constitute a significant contribution to the culture of mathematicians or physicists. Of more interest for scientists are detailed studies of specific scientific achievements, in which they could observe concrete ways of doing successful science, and that could become a kind of model for their own research. It is well-known that the hypothetico-deductive method in mathematics for instance is a way of presenting results, but not a way to discover new results; the elements of method to discover new results can only be found by looking at concrete scientific works. To this, one could object that there are no such constant laws in the scientific method, as has been shown for example by P. Feyerabend. ${ }^{4}$ This is true, in a way, but only if one understands that these laws should be, like scientific laws, applicable everywhere in the exact same way. On the contrary, the laws of method are, like the laws of human condition that Thucydides wanted to illustrate, much closer to civil laws: they have to be adapted to the case at hand. It is precisely because such laws cannot be stated universally that scientists have to invent them, and it is precisely because they are hard to invent that one should better, whenever possible, adapt them from a solution to a similar problem.

Declaring that history is a source of inspiration is thus very similar to G. Pólya's advice to students to look back when they have found a solution to a problem: "By looking back at the completed solution, by reconsidering and reexamining the results and the path that led to it, they could consolidate their knowledge and develop their ability to solve problems." " If this kind of reflexivity is beneficial for a student, then there are no reasons to believe that it is not, on a larger scale, also helpful for advanced scientists.

3 Thucydides, History of the Peloponnesian War, I, 22, 4.

4 Feyerabend, P., Against Method.

5 Pólya, G., How To Solve It, I, § 13, pp. 14-15. 


\subsection{History as a Condition of Progress}

The perhaps most used quotation to justify the need of history is G. Santayana's aphorism: "those who cannot remember the past are condemned to repeat it." It is, however, often given a different meaning than the one Santayana had in mind when he wrote it down. Because it uses the verb "condemn," it is interpreted as referring to past errors only, and indeed it seems that their repetition is better prevented when they are known and documented. This interpretation would give a rather limited and negative role to history, namely, to record the wanderings of the past. Its original meaning becomes clear by looking at its immediate context: "Progress, far from consisting in change, depends on retentiveness. When change is absolute there remains no being to improve and no direction is set for possible improvement; and when experience is not retained, as among savages, infancy is perpetual. Those who cannot remember the past are condemned to repeat it." In other words, Santayana does not tell us that it is necessary to remember past mistakes to avoid falling in the same traps, but that it is necessary to retain experience in order to progress; if not, then knowledge or society stagnates.

The need of history does not, however, follow immediately from this observation: even in sciences, in which studying original documents is currently not seen as something important, experience is retained, but it is absorbed and synthesized in later works. There is thus no risk to "reinvent the wheel," at least for the most important results. However, something is lacking in such syntheses, namely what the now common interpretation of Santayana's aphorism designates: the ideas that seem good only for a while, the failed attempts to solve a problem, the methods that have proven to be wrong. The risk is thus, in trying to solve the problem of the motion of vehicles, to reinvent something that is not a wheel. This is the second role of history, complementary to the first one: it can help scientists to detect early on that a path they could be tempted to take is actually a dead alley. Put another way, history can help them to walk faster on the paths of science.

\section{History: For Whom?}

History has, unlike other disciplines, no specific subject to study, except history of history. All other subjects that it could study already belong to another field of human knowledge. The immediate consequence of this fact is that the primary audience of historical works are never other historians, with the exception of history of history. In disciplines in which history is currently not seen as something important, it is thus tempting to target the layman, because of the lack of interest of the participants of the field. However, as we have just seen, history is, at its best, a source of inspiration and a condition of progress. This implies that the history of a field should, on the contrary, as much as possible be targeted at the primary actors of that field. This can be seen in the fields in which history already plays an important role: studies on Hegel's philosophy are written primarily for philosophers, and studies on Le Corbusier's

6 Santayana, G., The Life of Reason, vol. I, ch. XII, p. 284. 
architectural creations are written primarily for architects. Targeting the general public would inevitably imply to dumb the material down to reach the lowest common denominator of its interests, and therefore to lose the attention of those who are the main contributors to the field.

An additional difficulty, clearly not unique to the case of computing, arises at this point: the field of computing involves many different aspects, which are far apart from each other. Computer programmers, hardware designers, capital investors, computer scientists and computer users are all primary actors of the field, and yet their interests do not overlap and are even in conflict with each other. The kind of details and the kind of approach that will interest a computer scientist will probably not interest a computer user, and vice versa. History is, in that respect, very much like cartography: it is not possible to put every possibly relevant information on a single map; otherwise it becomes unreadable. Latitudes and longitudes, fauna and flora indications, roads and paths, buildings, geological or agricultural information, names, traffic density, altitudes, average temperature and precipitation, crime rates, population density and average wealth are for instance all relevant information, but only a few of these can be put on a given map, depending on its purpose. Even the scale of a map depends on its purpose: a too small one is impractical for car journeys, and a too large one useless for hiking. It would obviously be absurd to try to argue that one of these maps is better than the others: it all depends, again, on their aim. What is clear however is that its purpose should be explicit, and likewise that the target audience of a historical work should be clearly identified. Put another way, it is thus necessary to make a distinction between the different audiences, because it is impossible to address all of them simultaneously. The resulting historical works will be very different depending on the chosen target audience, and will probably be mostly useless, except occasionally, to other audiences.

We will therefore briefly consider three possible audiences for history of computing: computer scientists $(\S 2.1)$, computer programmers $(\S 2.2)$, and teachers ( $\$ 2.3$ ). Other possible audiences could, and certainly should, be considered, but they fall outside of our area of least incompetence.

\subsection{History for a Computer Scientist}

Confronted with the idea that studying history could perhaps be useful for him, a computer scientist would probably immediately object that his science, and for that matter any other science, is concerned with truths that are independent of time and place, and not by the particularities of this or that specific past event. The four-square theorem for example was first stated by Diophantus during the third century, and was first proven by Lagrange in 1770 , but it would not change anything to mathematics if it was suddenly discovered that this theorem was already stated by Archimedes, or that Lagrange actually copied his demonstration from a not yet known manuscript of Fermat.

This radical opposition between the scientist's and the historian's approaches seems irreconcilable: the main task of a scientist is to establish general laws, whereas a historian should, on the contrary, refrain as much as possible from doing so. 
This opposition is so strong that Aristotle considered that even poetry is more universal than history. ${ }^{7}$ This binary view is not incorrect, but it misses something important, namely that science is a human activity, and that its results are thus discovered over time. This does not mean, obviously, that scientific laws are relative, but only that the order in which the different elements of a science are discovered is not without importance and without meaning. New concepts and theories are always introduced in presence of a difficulty: this implies that one cannot correctly understand the nature of a scientific concept if one does not see how it was born, that is, for what problem it was a solution, and what was the difficulty that it allowed to overcome. There is always a part of arbitrariness in scientific concepts, and understanding why and how they were created is necessary to understand them better, very much like understanding the motives of someone's choices is necessary to understand them. Such studies are thus necessary to become conscious of their arbitrariness: scientists are often so accustomed to them that they could tend to forget that they could have been different, very much like one tends to forget that counting in base ten or having weeks of seven days is arbitrary. Historical works written for computer scientists should therefore try to give an insight into the core concepts of the discipline, by trying to identify the origin of the difficulties that led to the creation of these concepts, and by comparing them with the other options that were rejected. ${ }^{8}$

Another argument that a computer scientist could object to the need of history is that the aim of science is to advance, and that history, looking essentially backwards, cannot, by its very approach, help a science to do so. This is, again, correct, but it relies on a too narrow understanding of what science is. Doing science does not only consist in piling new discoveries on top of an existing knowledge; it is also a matter of organizing this knowledge and these discoveries as a coherent whole. There are thus at least two different ways to contribute to the progress of a science: either by discovering a new result, or by putting order in it; history can help a scientist at least for the latter task. More generally, historical works can help scientists to put the latest discoveries in perspective, to determine to what degree a claimed novelty is actually novel, and to become more conscious that their discipline evolves slowly. In other words, it can help a discipline to constitute itself as a coherent body of timeless truths.

Finally, for a young discipline such as computer science, in which the fact that the underlying hardware changes so fast gives the inevitable impression that it is continuously evolving, history may also contribute to an increase of the perception of its stability over time, that is, to an increased consciousness of its scientificity.

\subsection{History for a Computer Programmer}

A programmer will probably be even less interested in historical studies than a scientist, at first sight. It is for example totally irrelevant, in the daily practice of a

\footnotetext{
Aristotle, Poetics, 1051b6-7.

8 An example of this kind of work, in a different field, is Bourbaki, N., Éléments d'histoire des mathématiques.
} 
doctor, that the stethoscope she uses has been invented by a Breton called Laennec in 1816; she will certainly not be a better doctor if she knows this. Likewise, it is not important for a programmer to know who discovered the sorting algorithm he uses, what language was the first object-oriented one, or who invented the principles of database organization. If history consists in documenting who invented what and when, then it is indeed useless for an engineer. What could, on the contrary, be interesting for engineers is a precise exposition of the intellectual conditions under which the techniques he uses were invented. Studying the context of the discovery of these techniques will not only help him to grasp their precise meaning and purpose: it also illustrates a way of approaching new problems that has proven to be successful. Acquiring this way of thinking is perhaps even more important for programmers than mastering these particular techniques.

Concretely speaking, historical studies aimed at computer programmers should take the form of detailed expositions of the implementation of innovative computer programs. Such expositions can help him to understand the thought processes of those who created them, and to get a better problem solving mindset. These expositions should not only present the implementation itself, but, as far as possible, the alternative schemes that were not chosen, along with the reasons why they were not chosen. Finally, because computer programmers try to avoid introducing errors in their programs, and put lots of energy in correcting them, such studies should also present the errors that were discovered, how they were discovered, why they were introduced, and how they were solved. ${ }^{9}$

\subsection{History for a Teacher}

A teacher is in a very different perspective than a scientist or an engineer: her aim, as a teacher, is not to discover new results or to implement new computer programs, but to present the most important results and techniques as clearly as possible to her students. It seems that for doing this she does not need to study history, because she can use the work of experts who have already spent time to digest the literature and to present it in reference textbooks. Using primary sources instead of textbooks would be a waste of energy, because she would redo something that has already been done, and most probably not as well. Moreover, the original presentation of many important results or techniques is often obscured by an outdated vocabulary, or by obsolete notations.

All this is true, but it considers the problem of teaching only from the point of view of the teacher and of the results to be taught. Equally important are, however, the student and his difficulties, and it is there that history can play a role. Studying original papers can help the teacher to understand why something that she considers obvious or trivial is actually not obvious or trivial at all: not only did it take years or centuries to develop, but it was considered difficult at some point, it was not

9 An example of this kind of work is Organick, E. I., The Multics System: An Examination of its Structure. 
immediately adopted, and it was perhaps even criticized when it was proposed. In short, there is no better way to become really conscious that something is not easy than to realize how much people have struggled with it.

However, such studies can only be profitable if one assumes that there is at least a similarity between the difficulties of the students and the difficulties experienced by those who discovered the results and techniques, or those who were first in contact with them. Such a similarity seems indeed to exist, and G. Pólya even draws a parallel between Haeckel's biogenetic law, which states that "ontogeny recapitulates phylogeny," that is, that the development of an individual animal mimics the evolution history of its species, and mental development compared to the development of a discipline. ${ }^{10}$ It is indeed clear that students will have, at the very least, the same difficulties as the thinkers of the past who had to discover the concepts and to master them: there is no reason to believe that a concept has become obvious simply because time has passed since it was discovered.

\section{History: How?}

Some elements about the kind of approaches that can be used in history of computing have already been given above, but it is now possible to characterize it more generally, albeit negatively, by pointing out three common temptations of historical research: focusing on "firsts" ( $\$ 3.1)$, exhaustiveness ( $\$ 3.2)$ and anachronism (§ 3.3).

\subsection{The Temptation of "Firsts"}

Chronologies are, admittedly, not much more interesting than listing the random draws of a lottery by date; they even put many schoolboys off history. The reason behind this fact is obviously not that establishing facts is not important for a historian, but that facts are a mere starting point of his work. What makes a historical work interesting and useful is not the collection of brute facts on which it relies, but the causal relations between these facts; the proper work of a historian is to uncover these relations.

One of the consequences of this rule is that history should not focus on the paternity of ideas (or, even worse, on the paternity of words), because determining who invented this or that or who deserves this or that title has little to do with the causal relations that should be sought. It is well known that results and ideas are often discovered independently by different people, sometimes after having been forgotten for some time, and that ideas rarely spring out of nowhere, but are often the result of a larger cooperation. It is thus far more enlightening to try to understand for what reasons someone became aware of an idea than to try to determine if nobody ever had that idea before him. For that matter, most debates on "firsts" are debates on definitions, which implies that they can have no conclusion.

${ }^{10}$ Pólya, G., The Teaching of Mathematics and the Biogenetic Law. 


\subsection{The Temptation of Exhaustiveness}

A possible difficulty of historical research, certainly present in history of computing, is the enormous amount of material that has to be dealt with. One of the effects of this fact on historical works is that one could be tempted, in the name of accuracy, to give as many details as possible and to give as many references as possible, resulting in a gratuitous show of erudition.

The similarity between history and cartography, of which we made use above, can again be helpful here: it is never possible to put all the details on a map and, even if maps of a smaller scale will contain more information than others, they will never indicate every plant, every stone and every lump of dirt. Likewise, one of the main tasks of a historian is to make a selection among the facts of which he is aware, that is, to identify the facts and references that are relevant to his topic, as in museums for example, in which only a tiny fraction of the collections are on display. Otherwise historical accounts become noisy and, in the end, unreadable, because they add an unnecessary burden for the reader, namely to select the relevant information in the vast amount of details.

\subsection{The Temptation of Anachronism}

Anachronism consists, in its most common form, in reading the past through modern glasses, that is, in interpreting the events of the past from the standpoint of the present. The consequence of anachronism is not only that it does not do justice to the past, but mainly that it prevents us to learn something from it, because our ideas are often foreign to those of the past and will inevitably affect its interpretation. For example, interpreting Euclid's Elements with modern algebraic symbols would be anachronistic, because he is reasoning with magnitudes and not with quantities; likewise, translating Aristotle's Analytics into the symbolism of modern logic would be anachronistic, because he is reasoning about concepts and not about symbols. The correct historical attitude, on the contrary, bears some resemblance to the method used by Husserl for instance, namely "to begin in absolute poverty, with an absolute lack of knowledge."11 This does not mean that the less the historian knows about the topic he studies, the better he will be, but that he should temporarily forget what he knows to enter the minds of the actors of the past without preconceived ideas.

An immediate consequence of this rule is that writing history by conducting interviews, which is especially tempting in history of computing because some of the main contributors to the field are still alive, should be avoided. It is certainly much easier to do than to dig patiently and attentively in the primary sources, and it will probably even be easier to read, ${ }^{12}$ but it will always result in a very biased picture of the past, and most probably in a history focusing on anecdotal details and accidental circumstances. It is well-known that memory distorts the past, which means that an

\footnotetext{
11 Husserl, E., Cartesian Meditations, $\S 1$.

12 See Thucydides, History of the Peloponnesian War, I, 22, 4.
} 
actor of a past event will, many years later, explain it with what he now understands, and with what happened after it, whereas a historian should try to explain that event only with what was understood and what happened before it. Simply stated, interviews are not reliable sources, and using the best available sources has always been a necessary condition of historical research. ${ }^{13}$ More generally, even carefully written recollections should be taken with an extreme care; it is only when the primary sources are not available anymore that it can be necessary, by lack of better information, to use secondary sources.

In the fields in which history already plays an important role, history is, by the way, never based on interviews or on recollections; these success cases should perhaps be taken as a model, before trying to invent a new way of doing history. One could of course object that the original documents are not free of errors, which is true. However, the fact that interviews are inherently unreliable sources does not imply that it is possible to rely on original documents without submitting them to a critical analysis; what is at least certain is that original documents are not affected by later ideas and facts. One could also object that the bias or the errors that can exist in a single interview could be compensated by working with collections of interviews from many different actors; alas, historical truth is not more democratic than scientific truth. $^{14}$

\section{Conclusion}

Paraphrasing Santayana, we can conclude that "those who can remember the past will repeat it." As we have seen, this does not mean that history seeks to resurrect the past, but that its main aim is to help the participants to a field to benefit from the experience of those who came before them. The best service that history can do for them is to provide them with detailed case studies, oriented towards their specific needs, that can become for them a source of inspiration. Following an old medieval saying, we could say that this can help them to become "dwarfs standing on the shoulders of giants." This saying has two complementary interpretations: its modern interpretation puts the emphasis on the fact that the dwarf can see a bit further than the giant, and its medieval interpretation on the fact that the dwarf is much smaller than the giant. ${ }^{15}$ In other words, history can be helpful both to progress, and to remain modest.

Acknowledgments. The author thanks K. R. Apt, H. Cazes, M. van Emden, M. Laurent, B. Le Charlier and P. McJones for their comments, suggestions and support.

13 See for example Thucydides, History of the Peloponnesian War, I, 21, 1, and I, 22, 2-3, and Ranke (von), L., Deutsche Geschichte im Zeitalter der Reformation, bd. I, p. ix.

14 Ranke (von), L., Deutsche Geschichte im Zeitalter der Reformation, bd. I, p. x.

15 Jeauneau, E., "Nani gigantum humeris insidentes" - Essai d'interprétation de Bernard de Chartres. 


\section{References}

Aristotle: Poetics (ante 322 B. C.)

Bourbaki, N.: Éléments d'histoire des mathématiques, Hermann (1984)

Feyerabend, P.: Against Method. New Left Books (1975)

Husserl, E.: Cartesian Meditations (1929), Cairns, D. (tr.). Martinus Nijhoff (1950)

Jeauneau, E.: "Nani gigantum humeris insidentes" - Essai d'interprétation de Bernard de Chartres. Vivarium 5, 79-99 (1967)

Kuhn, T.S.: The Structure of Scientific Revolutions. University of Chicago Press (1962)

Organick, E.I.: The Multics System: An Examination of its Structure. MIT Press (1972)

Pólya, G.: How To Solve It. Princeton University Press (1945)

Pólya, G.: The Teaching of Mathematics and the Biogenetic Law. In: Good, I.J. (ed.) The Scientist Speculates, Heinemann, pp. 352-356 (1962)

Ranke (von), L.: Deutsche Geschichte im Zeitalter der Reformation, bd. I. Duncker und Humblot (1839)

Santayana, G.: The Life of Reason. Charles Scribner's Sons (1905)

Snow, C.P.: The Two Cultures. The New Statesman and Nation 52, 413-414 (1956)

Snow, C.P.: The Two Cultures and The Scientific Revolution, 1959 Rede Lecture. Cambridge University Press (1959)

Thucydides: History of the Peloponnesian War (ante 395 B. C.), Mynott, J. (tr.) (The War of the Peloponnesians and the Athenians). Cambridge University Press (2013) 